\title{
Cambios en la realidad social: dinamismo y procesos de ajuste en el marco de los Servicios Sociales de Atención Primaria
}

\section{Changes in Social Reality: Dynamism and Adjustment Processes within the Framework of Primary Care Social Services}

\author{
Mar CERCADiLlo Isla y equipo ${ }^{1}$ \\ Mancomunidad de Mejorada del Campo-Velilla \\ mcercadillo@ssmejoradavelilla.org
}

Recibido: $19 / 06 / 2013$

Revisado: 27/06/20132

Aceptado 11/11/2013

Disponible on line: 20/12/2013

\begin{abstract}
Resumen
Este artículo parte de la experiencia de un centro de Servicios Sociales de Atención Primaria y de su reflexión sobre los cambios en las necesidades y las demandas de los usuarios/as ante las fluctuaciones sociales emergentes. Hemos intentado plasmar el proceso de equipo y cuál ha sido el transcurrir entre las causas de crisis, sus consecuencias en las personas, las familias y los grupos sociales, para acabar en la propuesta de líneas de intervención y estrategias de acción. En este caso ha sido tan interesante el producto final como el proceso para crearlo.

Palabras claves: Exclusión Social, Estrategias de Inclusión Activa, Crisis, Sistemas de Protección, Estado de Bienestar, Procesos Esenciales, Cambios Sociales.
\end{abstract}

\begin{abstract}
This article is based on the experience of a Primary Care Social Services center and its reflection on the changes in the needs and demands of clients faced with emerging social fluctuations. We have sought to capture the team process and what has taken place between the causes of the crisis and its consequences for people, families and social groups, to end with a proposal for areas of intervention and action strategies. In this case, the final product was as interesting as the process it took to create it.

Keywords: social exclusion, active inclusion strategies, crisis, protection systems, welfare state, essential processes, social change.

Referencia normalizada: Cercadillo Isla, M., et al. (2013): «Cambios en la realidad social: Dinamismo y procesos de ajuste en el marco de los Servicios Sociales de Atención Primaria». Cuadernos de Trabajo Social, 26(2): $265-274$.

Sumario: Introducción. 1. Proceso de creación. 2. Los escenarios sociales a partir de la situación del mercado laboral. 3. Factores compensadores: de las estrategias familiares a las estrategias comunitarias. 4. La exclusión social un fenómeno en continua evolución. 5. ¿Podemos combatir las causas de la crisis o atajar las consecuencias psicosociales? 6. Referencias bibliográficas.
\end{abstract}

${ }^{1}$ Los autores/as del presente artículo son: Aranzazu Campos, Trabajadora Social. Mar Cercadillo, Socióloga y Directora. Ignacio García, Mediador Intercultural. Alba Garcia, Trabajadora Social. Elvira Monreal, Trabajadora Social. Marta Montouto, Trabajadora Social. Victoria Mundi, Educadora Social. Feliciana Pérez, Trabajadora Social. Raquel Poderoso, Trabajadora Social. Anunciación Rodríguez, Trabajadora Social. Carmen Vallina, Trabajadora Social. Mancomunidad de Servicios Sociales «Mejorada-Velilla». 


\section{Introducción}

Los Servicios Sociales de Atención Primaria son un recurso cercano a la ciudadanía. La proximidad confiere a sus profesionales la oportunidad de conocer la realidad de primera mano, sentir los cambios sociales, poner cara a las dificultades y a los éxitos, escuchar las pequeñas voces con las que se construyen argumentos; estos y estas profesionales son los alquimistas capaces de transformar cada proceso esencial ${ }^{2}$ en una oportunidad, de conjugar recursos efímeros en respuestas válidas y en saber que la escucha activa puede ser un elixir para el alma.

Todo lo positivo que aporta la cercanía, en muchas ocasiones, nos lleva a quedar inmersos en la propia realidad. La falta de tiempo, la organización del trabajo, la prioridad de la atención sobre la reflexión no permiten, en todos los casos, ampliar el foco y ser capaces de analizar con mayor detenimiento los hechos sociales y proponer soluciones eficaces.

En el equipo de Servicios Sociales de la Mancomunidad «Mejorada-Velilla» ${ }^{3}$ existía la inquietud profesional de extrapolar la práctica habitual a la teoría; de habilitar espacios y tiempos para investigar, narrar y exponer las buenas prácticas que se obtienen en la intervención que realizamos, y que se producen en todos los centros de Servicios Sociales de Atención Primaria.

Escribir este artículo se ha convertido en una oportunidad para iniciar esta andadura. Nos ha permitido, en poco tiempo, empaparnos de los análisis de otros profesionales, ponerlos en común, construir una visión conjunta de lo que acontece, atribuir nombre a los fenómenos, elevar lo individual a lo colectivo, y lo más importante: proponer y planificar líneas de actuación para la intervención social, que quedarán reflejadas en este artículo y se materializarán en la práctica. Se convierte en una propuesta para la mejora en la actuación del Trabajo Social.

\section{Proceso de creación}

Concedemos parte de las letras que tintinean en este escrito a su propio proceso de creación, porque creemos que es posible que los Servicios Sociales de Atención Primaria no se escuden en la falta de tiempo, en la acumulación del trabajo, para no reflexionar de forma conjunta y no escribir las buenas prácticas ni trasladarlas a foros donde nos podamos nutrir de ellas otros profesionales.

\subsection{Metodología de la creación}

Para el abordaje de un nuevo trabajo de análisis es tan importante la recogida de información en el ámbito inmediato como nutrir el contexto y el marco teórico. Hemos elegido una amplia gama de textos actuales que en su mayoría se enmarcan en el enfoque de la Política social.

Para configurar la metodología hemos bebido de varias disciplinas, en particular del Trabajo Social y la Sociología. El Trabajo Social, a través de la entrevista, es capaz de recoger y atesorar una gran cantidad de información. La riqueza de todos los datos e historias de vida que obtenemos en la actividad diaria de los profesionales nos hace poseer una fuente enorme de conocimiento de la realidad. Unido a ello, decimos realizar entrevistas en profundidad a personas que utilizan el servicio para incidir sobre el modo como les afecta la crisis en su vida personal. Con ello priorizamos la voz directa a las conclusiones profesionales y a la literatura que configura el marco teórico, por encima de los criterios de representatividad sociológica.

\subsection{Elección y reparto de material escrito}

Si en el último siglo los cambios y la volatilidad de los procesos han sido una de las características principales, en este último lustro estamos expuestos a que cada mañana acompañemos nuestro café con una transformación que, en muchos casos, no somos capaces de digerir. Es-

\footnotetext{
${ }^{2}$ Procesos esenciales: son todas las interacciones con los usuarios. La esencia de estos procesos es la comunicación interpersonal. En torno a estas acciones se articulan los demás procesos de gestión de los Servicios Sociales (Junta de Castilla y León, 2004).

${ }^{3}$ La Mancomunidad de Servicios Sociales «Mejorada-Velilla» está compuesta por dos municipios de la Comunidad de Madrid: Mejorada del Campo y Velilla de San Antonio. Estas localidades se encuentran situadas en la zona periurbana del municipio de Madrid y albergan 33.000 habitantes.
} 
to supone que sea necesario estar interpretando constantemente la realidad; por ello la producción de estudios, documentos y artículos es muy prolífica. Lo que nos ha ayudado a tener un número ingente de documentación y de datos estadísticos.

\subsection{Planificación de las sesiones y objetivos}

Se realizaron tres sesiones, con una duración de dos horas. La minuciosidad en la explicación tiene por finalidad demostrar que es fácil integrarlo en el normal funcionamiento de un centro de Servicios Sociales.

En la primera sesión se volcó toda la información de los textos; detallando cuáles eran la ideas más relevantes de cada documento, llegando a configurar un mapa detallado de la situación social. Una orografía compuesta por el perfil de los grupos más afectados por la situación socioeconómica, por el discurrir de los sistemas de protección en nuestro país, el detallado relieve de la exclusión social y el clima desde la percepción grupal, además de ir apuntalando algunas hipótesis. Con toda esta información se configuró el perfil de las personas a las que íbamos a entrevistar y la estructura de la entrevista (trayectoria laboral, percepción de los cambios, potencialidades y demandas a los sistemas de protección):

- Parados/as de larga duración mayores de 45 años con cargas familiares; que hubieran desarrollado su actividad profesional en los sectores que han expulsado más trabajadores (construcción, transporte, hostelería, etc.). Se realizaron cuatro entrevistas, a dos hombres y a dos mujeres.

- Jóvenes menores de 25 años, con estudios acabados o en proceso, sin emancipar. Se llevaron a cabo dos entrevistas, a un hombre y a una mujer.

- Parejas jóvenes con hijos/as. Se realizaron tres entrevistas, a dos mujeres y un hombre.

Todas las personas entrevistadas tenían en común el ser usuarios/as de los Servicios Sociales. A algunos, la situación de crisis les había llevado a los Servicios Sociales y en otros casos ya habían sido acompañados por ellos en procesos vitales anteriores, pero ahora, su situación económica estaba en el centro de la intervención.

En la segunda sesión el objetivo era analizar los discursos de las entrevistas y unirlos al gran bagaje de información que atesoramos en los despachos. Pretendíamos aterrizar el macrodiagnóstico en la realidad de los municipios en los que centramos nuestra intervención.

En la tercera sesión se dio forma a las hipótesis que habíamos ido apuntando en las sesiones anteriores e hilvanamos las líneas de actuación. Como a lo largo del desarrollo del artículo se irá apuntando, no hemos podido evitar indicar qué creemos que se debería cambiar o reforzar en los sistemas de protección social del un Estado de bienestar cada vez más mermado como el nuestro. No obstante nos hemos centrado en lo que la experiencia nos ha otorgado y en los ámbitos en los que creemos y sabemos que somos expertos: la familia, la intervención grupal para el emprendimiento y el refuerzo de las redes sociales.

Adelantamos una conclusión: la insignificante permeabilidad entre la configuración de las políticas sociales y el Trabajo Social de base. Como apunta Orlanda Díaz-García (2012), el alcance de las habilidades del Trabajo Social y lo extenso de su aplicación es una buena base para el análisis y la implementación de nuevas políticas sociales.

\section{Los escenarios sociales a partir de la si- tuación del mercado laboral}

Numerosas opiniones califican de histórico este momento. Posiblemente estemos a las puertas de un cambio profundo de sistema, que ya no da repuestas válidas a la realidad con la que convive. Pero más allá de las suposiciones, lo que se puede afirmar es que los hechos no son nada halagüeños. Lo que mostramos a continuación es una panorámica de la situación socioeconómica, que nos ha ayudado a trazar estrategias profesionales que son necesarias para la inmediata intervención.

Uno de los problemas más acuciantes de la crisis en España es la elevada tasa de paro (Ministerio de Empleo y Seguridad Social, 2013) ${ }^{4}$. A este aspecto hay que sumar que el gran pilar

${ }^{4}$ El 25 por ciento para España, el 20 por ciento Comunidad de Madrid y el 15 por ciento en la Mancomunidad. Datos para el segundo trimestre del 2013 del Ministerio de Empleo y Seguridad Social. 
de la protección social en nuestro país es la Seguridad Social, por lo que la cobertura individual y familiar se mide por la aportación a ésta a través de las retribuciones del trabajo y el tiempo de actividad. Por esta razón la situación se muestra tan perversa y tiene consecuencias tan devastadoras:

Los jóvenes presentan la tasa más alta de paro, un 57 por ciento, siendo el 51 por ciento para la Mancomunidad que supone el 11 por ciento de la cifras de paro registrado en el segundo trimestre del año (ídem). En la mayoría de los casos no han accedido al primer empleo y los que lo han hecho no tienen cobertura por desempleo, porque su cotización ha sido insuficiente. Las consecuencias inmediatas son un retraso importante en la edad de emancipación y la previsible emigración de la población más joven y cualificada en busca de alternativas de futuro.

Uno de los grupos más afectados por la crisis es el de las familias jóvenes con hijos. Suelen integrarlo personas que formaron parte de un mercado en expansión, pudieron emanciparse, accedieron a una vivienda - con un precio muy por encima de su valor y con una carga hipotecaria significativa - y formaron una familia. Ahora, se encuentran inactivos y con subsidios de desempleo que no compensan los gastos. Una de las consecuencias más importantes es el aumento de la pobreza y la exclusión infantil. Según los datos de UNICEF (2012) hay 2.200.000 niños en situación de exclusión social. La infancia es un sismógrafo que mide el estado de un territorio.

En una situación similar o aún más preocupante, se encuentran las familias monomarentales. Antes de 2007 eran las familias con más dificultades económicas; las mujeres, en el índice general, tenían empleos con menor retribución económica y la obligada conciliación familiar les abocaba a tomar medidas como asumir jornadas reducidas o medias jornadas.

Por otro lado también están las personas inmigrantes. La inmigración se ha considerado un fenómeno que se explica exclusivamente por factores económicos; por lo tanto se considera bajo una única dimensión, la condición de trabajadores de sus miembros. Con esta con- ceptualización se encuentran en los índices estadísticos más precarios. Añadimos además que se han endurecido los requisitos de acceso a las prestaciones y sistemas de protección, lo que incrementa la inequidad social.

Las personas consideras paradas de larga duración - que llevan al menos un año sin empleo- están distribuidas por todo la pirámide de edad. En todos los casos, encontrar empleo es un reto, pero para los mayores de 45 años (el 37 por ciento de las personas paradas de la Mancomunidad (ídem), con vidas laborales amplias y experiencia en un solo sector, se convierte en una casi imposibilidad,ando no escasez, de incorporarse al mercado laboral. Si sumamos el atraso en la edad de jubilación y la ampliación de los años cotizados a la Seguridad Social, el resultado es la desprotección de un sector de la población de grandes dimensiones.

Podemos asegurar, con sólo estas pequeñas pinceladas de realidad, que hay un número cada vez mayor de personas y familias en situación de exclusión. Pero afirmamos que las personas que se encontraban en esta situación antes del 2008 continúan en ella y en situaciones de mayor precariedad, con lo que estamos ante la cronificación de la pobreza social (Laparra y Pérez, 2012).

Por dar una nota de color indicaremos que, en términos absolutos, las personas mayores de 65 años no han perdido su capacidad económica con respecto al inicio de la situación de crisis. Y son ellas quienes se están convirtiendo en los verdaderos proveedores de protección social $^{5}$. Volver a residir con la familia de origen -los hijos boomerang (Instituto Internacional de Estudios sobre la Familia, 2011) - es una de las estrategias para capear la situación económica adversa.

Igualmente se asegura que el desempleo ha afectado más a los varones que a las mujeres. Algunas razones que explican esta menor afectación apuntan a que se hallan en sectores menos aquejados por el tsunami económico aomoí y por la compensación motivada por la inversión que las mujeres han hecho en formación, aunque, como ya se ha indicado cuando hacíamos referencia a las familias monoma-

${ }^{5} 300.000$ familias españolas viven de la pensión de una persona mayor de 65 años (Laparra, M. y Pérez, B., 2012) 
rentales, su situación ya partía con un escenario caracterizado por la desigualdad, y este dato no ha cambiado.

Por otro lado el extendido empleo de baja calidad, con sueldos pequeños y poca estabilidad, genera lo que se denomina «trabajadores pobres» —working poor-, personas que con su remuneración salarial no pueden hacer frente a los gastos básicos.

\section{Factores compensadores: de las estra- tegias familiares a las estrategias comuni- tarias}

\subsection{La familia}

Algunas de las conclusiones extraídas de las entrevistas revelan que, en los países mediterráneos, la familia ha sido el sistema social de protección: un modelo de familia con intensos lazos de apoyo, que ha dado cobertura a sus miembros de forma extensa y con una relación muy fuerte entre generaciones. A esta característica hay que añadir que los roles de género tradicionalmente establecidos han continuado en el tiempo; las mujeres han sido proveedoras de bienes sociales y han mantenido aquellas redes de cuidado que no generaba el Estado, quizá porque no existía tal demanda o por una falta absoluta de previsión. Incluso tras la eminente incorporación de las mujeres al mercado laboral y el consecuente reclamo de una diferente distribución de las tareas del cuidado, no ha habido una respuesta contundente ni por parte de las instituciones ni del sistema familiar. Las mujeres han seguido asumiendo una doble o triple tarea, la personal, la familiar y la laboral.

Este modelo familiar tan arraigado está siendo un factor compensador en la crisis. Nos encontramos con un amplio catálogo de modos de autoayuda: como suministradores de alimentos, otorgadores de becas a los menores para el material escolar, sufragadores de cuotas para evitar el endeudamiento, compartidores de viviendas y a partir de aquí, de la vida. Pero la familia no puede ser el único factor compensador, porque la duración de la crisis está llevando a un debilitamiento de la red familiar en la función protectora de sus miembros (Alaya, 2012).
Al final, la familia no es sólo proveedora de recursos, sino el escenario donde se tejen las relaciones afectivas. Es importante señalar cómo influye la situación socioeconómica en la gestión de las emociones y en la cohesión familiar.

Hemos identificado como los varones, que se han construido socialmente bajo los parámetros del espacio productivo, están protagonizando una mayor sensación de exclusión social. No poseer un empleo supone: no ser la figura abastecedora de bienes para la familia; debilitar o anular unas relaciones personales que se han construido alrededor de los espacios productivos, y no participar en foros sociales anexos a su actividad laboral. Como consecuencia, nos encontramos con personas que pasan por ciclos de desánimo ${ }^{6}$, que en algunos casos provocan diagnósticos clínicos de salud mental. Se parapetan en el silencio, en la desesperación y en la pérdida de un futuro cierto. Las mujeres, en circunstancias semejantes, también pasan por estados de ánimo parecidos, pero su preocupación explícita son sus hijos y cómo sacarles adelante. Son las que manejan unas redes que tejen con mucha imaginación, para ahorrar, reutilizar y sacar de donde no hay para llevar a su familia adelante.

No son pocas las personas que afirman que la coyuntura en la que se encuentran está deteriorando su relación de pareja. Igualmente surge el enfrentamiento con los hijos e hijas. En las escuelas de familias hay una opinión generalizada sobre el despotismo de los más pequeños e igualmente sobre la sobreprotección que los adultos ejercen sobre ellos. Esta característica tan extendida conlleva a que en este momento haya niños y adolescentes que no entienden la privación material a la que están sometidos o que sus progenitores hagan lo posible para que no se enteren y lleguen al endeudamiento para seguir manteniendo una burbuja familiar.

Como se ha indicado, una estrategia cada vez más extendida es la convivencia compartida con otros miembros de la familia. En la mayoría de los casos, se producen sentimientos encontrados: agradecimiento e incomodidad por tener que optar por una solución no voluntaria. Volver al hogar primigenio siempre tiene un regusto a fracaso y puede que

\footnotetext{
${ }^{6}$ Ciclo del desánimo: término acuñado por el equipo de Servicios Sociales «Mejorada-Velilla»
} 
también lleve a una confrontación generacional. La obligada adaptación puede generar conflictos que requieren apoyo profesional. Pero para realizar un diagnóstico certero tenemos que apuntar que las nuevas familias que llegan a Servicios Sociales poseen potencialidades: han sido resolutivas, han propiciado la evolución positiva de sus miembros y han utilizado una relación afectiva no dañina; son conocedoras de la realidad en la que viven y del límite de los recursos. Son familias con problemas, pero no son familias multiproblemáticas ${ }^{7}$. Por ello la intervención de los Servicios Sociales tiene que ser diferente: a partir de las oportunidades.

Para algunas familias, los cambios han supuesto un giro «positivo». La falta de actividad productiva no ha supuesto la inactividad social. Para algunos hombres el desempleo les ha ofrecido poder disfrutar de un tiempo personal y familiar que nunca habían poseído; si sus compañeras sentimentales trabajan, son ellos los que realizan las tareas del hogar y han tomado el protagonismo del cuidado; y lejos de analizarlo como una carga, están disfrutando de esta nueva etapa y valorando la importancia del trabajo reproductivo.

El discurso recogido en las entrevistas es doloroso porque sienten que no hay una solución cercana y se extiende un gran sentimiento de injusticia ${ }^{8}$; pero a la par, esas mismas personas, generan esperanzas y dan soluciones.

\subsection{La creatividad y nuevos hábitos}

Se está propagando una tendencia maliciosa que consiste en convencernos de que los recursos financieros y materiales no son importantes ni necesarios y que la imaginación y la creatividad suplen el resto de las carencias. No, esta corriente de pensamiento puede llevarnos a extremos como el de no asumir las responsabilidades que se esperan de un Estado benefactor y poner en manos de la individualidad los éxitos sociales.

Aun así, hay que destacar que las familias ponen mucha imaginación y creatividad para adaptarse a las nuevas circunstancias. El consumo ha descendido notablemente. En términos generales, es probable que este dato no sea positivo, pero se ha controlado el consumo innecesario. La reutilización se ha convertido en una práctica habitual, sobre todo en el uso de la ropa y de otros bienes personales no perecederos. Poseer tiempo ha rescatado una vieja práctica: la comparación de precios y la trashumancia entre los establecimientos. Se ha restringido el uso del automóvil —un dato positivo es el descenso de las emisiones de $\mathrm{CO}_{2}$ Se discierne entre lo necesario y lo prescindible. Pero también hay otros datos menos alentadores:

- La reducción del consumo de gastos fijos, luz, gas, etc., lo que supone que el invierno 2012-2013 ha habido un 28 por ciento de hogares, considerados pobres energéticos, cuyas viviendas han estado por debajo de 18 grados.

- La descapitalización. Las familias se ven abocadas a vender sus propiedades o a consumir sus bienes mobiliarios para protegerse de los vaivenes económicos.

- El endeudamiento. Han dejado de realizar pagos necesarios, agotando los recursos.

\subsection{La respuesta de los sistemas de pro- tección}

El Estado de bienestar es un conjunto de instituciones o sistemas proveedores de políticas sociales dirigidas a mejorar las condiciones de vida y a facilitar la integración de clases y grupos sociales más desfavorecidos o vulnerables. Un Estado de bienestar fuerte posee instrumentos para hacer frente a la exclusión social, pero esta crisis ha destapado una realidad incontestable: su gran insuficiencia.

Volvemos a señalar que la Seguridad Social y la aportación a ella es la que proporciona una parte de la protección social efectiva. En España no se han puesto en marcha otras políticas como las de apoyo a la familia y a la conciliación. No se han tomado medidas garantistas para las personas que no puedan tener acceso

\footnotetext{
${ }^{7}$ Familias en que el comportamiento sintomático funciona como un elemento de dificultad y es un disgregación añadida para el comportamiento de los otros miembros del sistema y para éste considerado en su globalidad (Cancrin, de Gregorio y Nocerino, 1997).

${ }^{8}$ Somos el quinto país de la Unión Europea con la percepción más pesimista sobre la realidad social (Laparra y Pérez, 2012).
} 
al mercado laboral, ni se han revalorizado ni regulados los salario mínimos de inserción. Todas ellas son medidas recomendadas para nuestro país en la Agenda 2020, en el marco de las políticas de inclusión activa. Bien es cierto que poseíamos dos sistemas públicos muy arraigados y con un excelente funcionamiento: el sanitario y el de educación. Pero las embestidas de la crisis están suponiendo un claro revés a estos dos grandes pilares.

En cuanto a los Servicios Sociales, el cuarto gran pilar del Estado del bienestar, tendría que haber sido reforzado con anterioridad a la crisis, pero no fue dotado en demasía y, en la actualidad, cuando más se le exige, menos recursos puede ofrecer. Los profesionales de Servicios Sociales somos muy críticos con nuestro propio sistema y con el carácter subsidiario que se le ha conferido. Vemos las limitaciones y la falta de recursos, pero es ahora más que nunca, cuando se acrecienta el valor de los recursos humanos y su profesionalidad.

Una de las características de los Servicios Sociales es la apuesta por «lo humano» y por todos los valores que le acompañan. Hace doce años la Mancomunidad realizó una investigación, entre los aspectos que se analizaron resaltaba cómo nos veía la ciudadanía. Se recogieron las mismas cualidades que se destacan en el pequeño diagnóstico que se ha llevado a cabo para escribir este artículo: la escucha activa, la comprensión, la empatía, la calidez, el asesoramiento, el apoyo y el acompañamiento. En definitiva: la proximidad.

Hemos de tener claro cuáles son las posibilidades, pero la profesionalidad y la metodología del Trabajo Social, en el sentido amplio del término, es su gran potencialidad

\subsection{Iniciativas social o comunitaria}

Debemos mencionar que la iniciativa social se organiza cada día para llevar a cabo acciones que mejoren la calidad de vida o para defender los derechos de otras personas o grupos. En la mayoría de los casos son igual de anónimos quienes los apoyan como quienes reciben el apoyo. Lo que vertebra la relación entre estas personas es la solidaridad y la participación social, cuyo objetivo es el bien común.

Podemos destacar aquellos movimientos ciudadanos que han conseguido ser muy mediáticos (STOP desahucios, por ejemplo),co- mo los pequeños movimientos de barrio que tejen redes para apoyar a personas vulnerables en su vecindario o aquellos profesionales que generan ideas o prestan sus servicios gratuitamente, implicándose de forma activa en estos entramados ciudadanos. Las redes sociales se han fortalecido. Los nuevos movimientos sociales han dejado de ser estatutarios y con objetivos a pequeña escala, para convertirte en solidarios y espontáneos, pero no por ello menos reflexivos.

\section{La exclusión social un fenómeno en con- tinua evolución}

Para los Servicios Sociales poner cara y voz a la descripción anterior es fácil, porque cada día cruzan la puerta de nuestros centros hombres y mujeres que narran en primera persona situaciones que hoy sintetizamos aquí como fenómeno sociológico. Tanto en las aportaciones que hemos recogido en las entrevistas específicas, como en las entrevistas personales que se llevan a cabo en los despachos, encontramos las demandas explicitas e implícitas de muchas personas. Lo acuciante, lo que nos genera un desconsuelo inmediato, es la solicitud de las familias para cubrir sus necesidades básicas. Pero esto tiene un término finito y la intervención social no puede pararse en el suministro de prestaciones de emergencia.

Con estas medidas no se ataja la pobreza, pero menos aún la exclusión social, es decir, un término de mayor amplitud contra el que combate el Trabajo Social. Se podría afirmar que la no inclusión o integración social no se mide tan solo por la falta de ingresos y el alejamiento del mercado laboral, sino también por un debilitamiento de las redes sociales, por el descenso en la participación social y la consiguiente pérdida de derechos sociales (Laparra, 2012).

El concepto de exclusión social vertebrado desde los tres ejes - económico, social y ciudadano- no es novedoso y se recoge en el Tratado de Lisboa (2000), que aporta recomendaciones para los países miembros de la Unión Europea, por lo que se refiere a las denominadas estrategias de inclusión activa.

\subsection{Estrategias de inclusión activa}

Las estrategias de inclusión activa promueven la participación social y laboral en sentido 
amplio: propician una renta adecuada, la inclusión laboral o social y el acceso a servicios de calidad. Por lo tanto las recomendaciones van dirigidas a que los mercados de trabajo sean inclusivos, estables y de calidad; que las personas que no puedan acceder al mercado laboral también posean una renta adecuada: las denominadas rentas mínimas de inserción; que cuenten con apoyo a la Dependencia o a la conciliación de la vida personal, familiar y laboral; y por último, una optimización del acceso a los servicios del bienestar, cuyo rasgo dominante son los avances en la coordinación entre los servicios de empleo, de formación y Servicios Sociales, tratando de establecer rutas y conexiones entre todos ellos (Rodríguez, 2012).

En España se aconseja que se simplifique y se agrupen los sistemas de protección o las garantías de mínimos, así como que se mejore la intensidad protectora de las rentas mínimas de inserción y se incentive la protección a las familias con menores. Por otro lado, se propone realizar un esfuerzo en la formación y en su seguimiento: conectar de forma real la educación y el aprendizaje con el entorno laboral, haciendo especial hincapié en la inclusión de los colectivos más alejados de los recursos de empleo. Para finalizar, se busca mejorar la calidad, ya existente, en los servicios: reforzar los servicios de empleo; establecer una intensa y eficaz coordinación entre educación, servicios sociales y empleo; universalizar la sanidad, la educación y los servicios sociales y considerar la vivienda como un elemento crucial para la inclusión real.

La teoría y la lógica, tras el diagnóstico, de un pequeño equipo de Servicios Sociales coinciden con las plasmadas en los tratados de política social. ¿Por qué entonces no se toman decisiones que se encaminen a la consecución de estos objetivos? Algunas de las estrategias necesitan presupuesto, pero otras sólo necesitan una gobernanza que pongan su mirada en el medio y largo plazo.

\section{5. ¿Podemos combatir las causas de la crisis o atajar las consecuencias psicosociales?}

\subsection{A modo de conclusión}

Con todo lo expuesto, la realidad se muestra como un gran puzle de diminutas piezas y es necesario ensamblar cada fragmento para vislumbrar las imágenes con nitidez. Así se plantea la construcción de la praxis; ya en el proceso nos vamos dando cuenta de que hay soluciones fuera de nuestro alcance, pero otras son factibles y es nuestra responsabilidad acometerlas.

Los Servicios Sociales de Atención Primaria, como hemos indicado, poseen una gran potencialidad: sus profesionales, pero también su metodología de trabajo centrada en las personas y las familias y en su acompañamiento; además de un gran bagaje en la intervención familiar con enfoques sistémicos, y en el trabajo comunitario, incentivando la participación activa y la coordinación interdepartamental. Estas son nuestras herramientas.

Hemos detectado las potencialidades y las posibilidades de la población de los municipios en los que desarrollamos nuestra intervención: la familia como factor de compensación, el refuerzo de las redes sociales, tanto las conocidas como las anónimas; la creatividad personal y social; el deseo de salir de la situación de estancamiento; el cambio en los valores y el considerar la formación como un elemento importante para salir de la crisis. Estas son sus herramientas.

También hemos señalado factores menos alentadores. Estos son ámbitos en los que emplear sus herramientas y las nuestras:

— Los «ciclos del estado de ánimo» por los que pasan las personas en situación de exclusión social, provocados no solo por la restricción en los recursos económicos sino también por la merma de las relaciones sociales y por el menor acceso a los servicios públicos o a los ámbitos de decisión. La sensación de falta de utilidad conlleva la depreciación del ser social. La pérdida del estatus social supone la desaparición de sus estructuras. Como hemos apuntado, influye más en los varones que en las mujeres.

- Las familias son un factor de compensación y apoyo, pero también son el lugar donde se producen las conexiones entre sus miembros. Las situaciones adversas conllevan que la estructura familiar pueda debilitarse o ceder: en la relación con los hijos, en la relación con la pareja y en los roles que se establecen entre ellos. 
- Cambio de roles de género: las políticas de igualdad han adolecido de un acercamiento igualitario entre varones y mujeres. Se pueden señalar algunas causas: la igualdad basada en el acceso al mercado laboral, la falta de políticas de conciliación real y la consiguiente falta de tiempo para implicarse en la vida familiar. Ahora tenemos tiempo y un mercado laboral que discrimina, pero sin atender a razones de género. Por lo tanto el cambio del sistema productivo puede suponer un cambio en los roles. Pensamos que este tránsito necesita un acompañamiento, ya que es necesario la valoración real del ámbito familiar: la participación en los ámbitos de la crianza, la provisión de servicios de autoayuda familiar y las denostadas tareas del hogar.

\section{2. ¿Dónde podemos intervenir y cómo? Propuestas de trabajo.}

Transformar el análisis en acciones, a veces es un salto cualitativo difícil de abordar; supone un nuevo reto para el equipo: articular propuestas, tras el análisis de la realidad y las conclusiones finales. A partir de la reflexión conjunta se generan las siguientes líneas de actuación que se pondrán en marcha en la planificación anual:

- Trabajo grupal con los varones: Nos parece importante el proceso grupal para que los varones puedan verter sus opiniones e identificar los nuevos parámetros sociales. La dinámica grupal acerca puntos de vista y muestra soluciones conjuntas.

- Intervención grupal con parejas: en la misma línea, es interesante que varones y mujeres se confronten, en una situación de equidad, lo que supone la asunción de roles igualitarios.

- Unir potencialidades y trabajar «los ciclos del estado de ánimo»: hemos podido comprobar que las personas que están en una situación de desempleo, pero que tienen una integración social positiva, no caen estados de desánimo porque poseen otros espacios de utilidad. Nos parece posible que las personas trabajen sobre sus potencialidades. Potencialidades que pueden haberles sido útiles en su vida laboral o que son habilidades que se encuentran por explotar. Para que sea más enriquecedora y creativa la intervención tiene que ser grupal, trabajando la relación entre las personas y la sinergia de ideas.

- Reforzar las escuelas de familia: En nuestro centro de Servicios Sociales se realiza un programa anual de escuelas de formación dirigidas a familias, pero consideremos de interés insertar de forma transversal la situación actual para que las familias reflexionen como está influyendo el cambio de paradigma en la relación entre padres y madres e hijos: una generación con un futuro incierto, cómo ha de convivir con hijos adultos, así como abordar el duelo del nido lleno, las relaciones intergeneracionales extensas, etc.

- Reforzar los programas de familia: si bien la opción de la intervención grupal es la línea por la que apostamos, sabemos que hay dificultades familiares que deben ser tratadas desde ámbito terapéutico. Una intervención no tiene sentido sin la otra.

- Trabajo grupal para información, asesoramiento y acceso a los recursos: para los Servicios Sociales de Atención Primaria es primordial que la primera cita sea personalizada, que se establezca el primer vínculo con el o la profesional. Pero hay demandas y necesidades que se reciben en los despachos que pueden ser tratadas en grupo. Se dimensiona una atención más rica y diversa. Con el trabajo grupal y en red estamos ofreciendo un modelo de ciudadanía.

- Apostar por la coordinación institucional y el trabajo en red: es imprescindible para dar respuestas a los cambios sociales propiciar la coordinación institucional y la interacción con organizaciones privadas. Se hace indispensable para acompañar los procesos grupales que seamos capaces de integrar un gran número de recursos. En la línea que apuntan las estrategias de inclusión activa, tenemos que incrementar el trabajo en red entre los servicios con el fin común de evitar las situaciones de exclusión. Los Servicios de Atención de Primaria tienen experiencia en liderar procesos de organización institucional y comunitaria y de abanderar servicios de calidad.

Ilusionar a otras personas, aprovechar la experiencia que atesoramos, utilizar la memoria y poner en valor la profesionalidad no ha de ser complicado. Son retos que nos hacen caminar y promover cambios. 


\section{Referencias bibliográficas}

Ayala, L. (2012). Los indicadores sociales. Exclusión y Desarrollo Social, 5-22.

Cancrini, L., De Gregorio, F. y Nocerino, S. (1997). Las familias multiproblemáticas. En M. Coletti y J. Linares (eds.), La intervención sistémica en los servicios sociales ante la familia multiproblemática. Barcelona: Paidós.

Colectivo IOE. (2011). Efectos sociales de la crisis. Una evaluación a partir del Barómetro social de España. Papeles de relaciones ecosociales y cambio global, 113, 177-188.

Colectivo IOE. (2012). La participación ciudadana. Algunos apuntes a partir del Barómetro Social de España. Cuadernos de Trabajo Social, 25 (2), 309-319.

Díaz-García, O. (febrero, 2012). El Trabajo Social ante la crisis económica. BIBLID, [on line] (XII - extra), 239-244. Disponible en: http://revistas.uca.es.

Family watch, the. (2012). El desempleo juvenil en tiempo de crisis y sus consecuencias. Disponible en www.thefamilywatch.org .

González Gago, E., Fernández Prat M., Laparra, M. y Pérez, B. (2009). Políticas de inclusión activas en el plano local (pp. 7-117). Cuaderno Europeo 6: Madrid: Fundación Luis Vives.

González-Bueno, G. (2012). La infancia en España, 2012-2013. Madrid: UNICEF.

Junta de Castilla y León. (2004). Trabajando con los procesos: guía para la gestión de procesos. Disponible en: ttp://www.jcyl.es/web/jcyl/AdministracionPublica/es/

Plantilla100DetalleFeed/1248367026092/Publicacion/1181202930899/Redaccion.

Laparra, M. y Pérez B. (coords.) (2012). Crisis y fractura Social en Europa. Causas y efectos en España. Barcelona: Obra Social La Caixa.

Lluch, E. (julio-septiembre, 2010). Consecuencias económicas y sociales de la crisis mundial. Revista de Estudios Sociales y de Sociología Aplicada, 158

Red Europea de lucha contra la pobreza y la exclusión social en el Estado Español. (EAPN-ES) (2009). El impacto de la crisis en la pobreza y la exclusión social: Diagnóstico y actuaciones. Madrid: Fundación Tomillo, EAPN-ES.

Rodríguez, G. (2012). Estudio de casos sobre estrategias de inclusión activa en países de la UE. Madrid: Ministerio de Sanidad, Servicios Sociales e Igualdad. 